\title{
Infrared reflectance measurements of thin films with time variable surface roughness or texture
}

\author{
C. Hoof, D. Wetzlar, B. Henning \\ University of Paderborn, EIM-E-EMT, Measurement Engineering Group, Warburger Str. 100, 33098 \\ Paderborn, Germany \\ hoof@emt.upb.de
}

\begin{abstract}
For contactless moisture measurement the infrared reflectance measurement is often used. This noninvasive and fast measurement method is suitable for determining the moisture content of bulk materials or coatings and can be used for example to characterize drying processes of paints. The measured infrared radiation is dependent on absorption and scattering in the layer. Moreover, the temporal and local surface dullness (e. g. roughness) or texture influences the measurement results depending on diffuse and direct reflection. In this study an experimental setup which detects both the direct and the diffuse reflected radiation parts is realized. The setup consists of a FTIR- (Fourier Transform Infrared) spectrometer which is extended for a simultaneous determination of spectrograms at different incidence angles $\left(0^{\circ}\right.$ as well as $\left.45^{\circ}\right)$.
\end{abstract}

Key words: moisture, FTIR-spectrometer, time-variable, dullness, PCA

\section{Introduction}

In a previous study for moisture measurement the radiation reflectance behavior was investigated using an experimental setup with only one fixed incident angel of $0^{\circ}$ [1]. The investigation showed clearly that the moisture measurand is additional strongly influenced by the surface properties (dullness, texture). To determine the moisture content, it is necessary to separate these effects.

\section{Concept}

This study deals with a new approach to determine the diffuse and the direct reflected radiation simultaneously (Fig. 1). Additionally, the detected radiation is influenced by absorption and scattering in the film (KubelkaMunk, [2] [4]). It is a typical situation in industrial applications that the surface roughness changes over time, for instance in due moved bulk materials (for example clay) or drying coat of paints. The transition from gloss to matt surfaces has here a special meaning.

The regular reflected radiation is significantly influenced by the surface texture or roughness. For this reason the reflected radiation is measured simultaneously at the angles of $0^{\circ}$ and $45^{\circ}$.

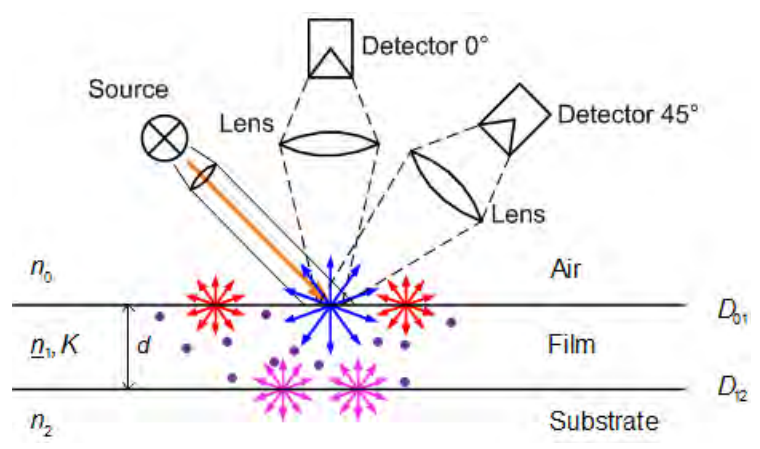

Figure 1: Principle of measurement: Detection of diffuse radiation at $0^{\circ}$, detection of regular and diffuse reflected radiation at $45^{\circ}$

\section{Experimental Setup}

To characterize the influence of surface roughness on the reflection behavior an arrangement consisting of broadband emitter and FTIR-spectrometer (Fourier Transform Infrared) is used (MIR 8000, see Figure 2).

The emitted radiation is modulated by a moving mirror in a Michelson interferometer. With knowledge of the mirror position the measurement results can be transformed (Fourier) into the spectrogram. The investigated spectral range depends on the spectral sensitivity of the used IR-detectors.

The experimental setup is arranged to measure the diffuse $\left(0^{\circ}\right)$ and the direct and diffuse reflected radiation $\left(45^{\circ}\right)$ simultaneously. The 
measuring object is a rough substrate moisturized with variable water quantities.

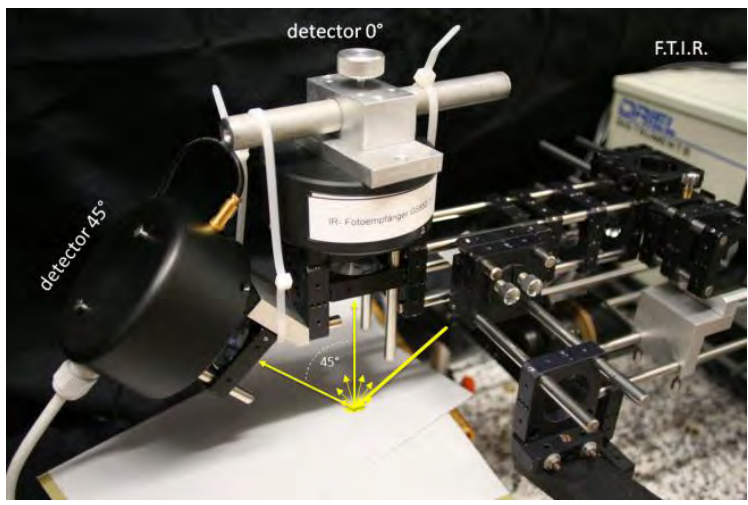

Figure 2: The realized measurement setup: The arrangement of the detectors at angles of $0^{\circ}$ and $45^{\circ}$ allows the detection of the diffuse and regular radiation components simultaneously.

An important goal of this study is to determine in detail the relation of diffuse and direct reflected radiation on the surface as well as the radiation absorption in the coating itself. The purpose is to measure the moisture even on gloss samples considering the disturbing influences.

Figure 3 shows the measured spectrograms at $0^{\circ}$ and $45^{\circ}$.
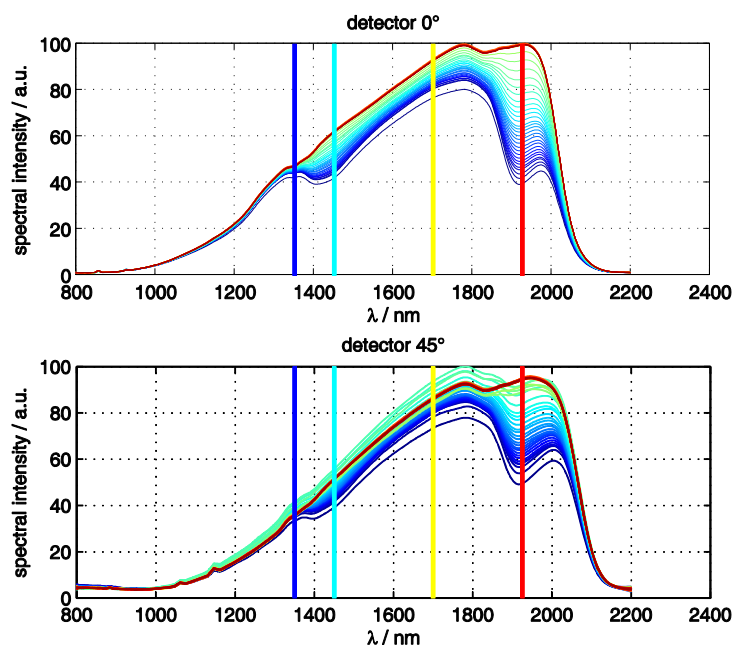

Figure 3: Normalized spectral intensities of a drying process on diffuse reflecting substrate, from blue (curves represent the initial state, wet surface) to green (dull finish) to red curve (dry surface). The four colored marker lines indicate discrete wavelengths considered in detail in figure 4.

At the beginning the wet surface is glossy and after the drying process the surface reflects mainly diffuse. In figure 3 the blue curves represent the initial state (wet surface) and the red curve the dry surface. In wet state the typical absorption bands of water can be seen in both plots. Figure 4 shows the measured spectral intensities at wavelengths $1,35 \mu \mathrm{m}$ and
$1,70 \mu \mathrm{m}$ (reference wavelength) as well as $1,45 \mu \mathrm{m}$ and $1,925 \mu \mathrm{m}$ (strong absorption of radiation caused by water) extracted from the FTIR-spectra (Figure 3) and referenced to their final value (dry surface).
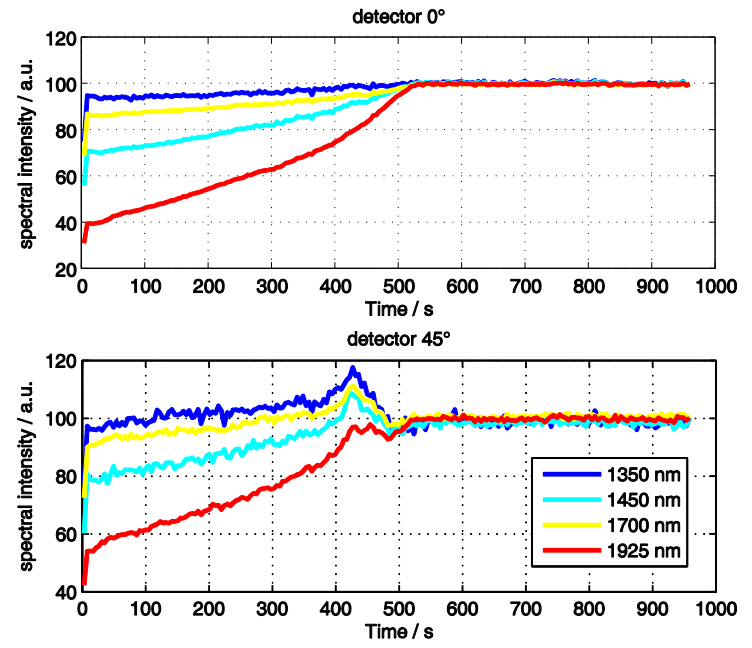

Figure 4: Measured and normalized spectral intensities at wavelengths $1,35 \mu \mathrm{m} / 1,70 \mu \mathrm{m}$ (reference wavelength) and 1,45 $\mu \mathrm{m} / 1,925 \mu \mathrm{m}$ (strong absorption of radiation caused by water), referenced to their final value (dry surface).

The radiation intensity at $0^{\circ}$ increases during the drying process. The detector captures only the diffuse component of the reflected radiation, which is significantly influenced by absorption in the layer. Additionally the detector signal slightly decreases by specular reflection.

The detector at $45^{\circ}$ captures the diffuse and directional radiation part. The detected radiation intensity is affected by absorption as well as reflections at the layer surface, which depends on the roughness of the surface (transition from matt to gloss).

First the intensity of radiation is low as a result of initially high absorption and multiple reflections in the water layer. The radiation intensity increases with decreased layer thickness caused by less absorption in the layer. The maximum of intensity is given at the transition to dull finish. The decrease of radiation intensity shows the diffuse reflective substrate is dry. 


\section{Qualitative analysis via PCA}

The measured radiation intensity is affected by absorption in the layer and effects caused by changed surface properties.

The PCA (Principle Component Analysis [3]) allows the extracting of the characteristics from large data amounts. In this case the measurement matrix $\boldsymbol{X}$ is established as follows. For $n$ points in time, a spectrogram is recorded with $m$ values. The measurement matrix $\boldsymbol{X}$ has the dimension $(m \times n)$ where $m$ is the number of observations at $n$ wavelengths.

$$
t_{\mathrm{i}}=X p, \quad i=1 \ldots n
$$

The first principal component $\boldsymbol{t}_{1}$ is obtained by transformation of the measurement matrix $\boldsymbol{X}$ with the first eigenvector $\boldsymbol{p}_{1}$ of the covariance matrix $\boldsymbol{X}$. The measurement matrix $\boldsymbol{X}$ is formed from the raw spectra to each of both detectors $\left(0^{\circ}\right.$ and $\left.45^{\circ}\right)$. The PCA is therefore applied to both data sets.

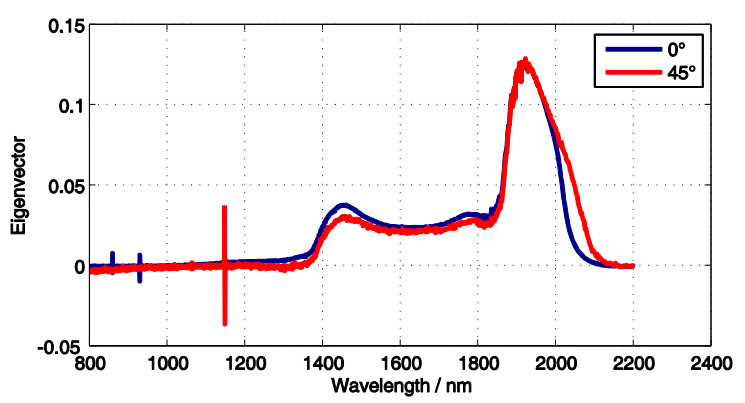

Figure 5: The first eigenvector of each raw spectra measured at $0^{\circ}$ and $45^{\circ}$

Both eigenvectors (at $0^{\circ}$ and $45^{\circ}$ ) show a very interesting result (Figure 5). They show the wavelength-dependent absorption of water weighted with the spectral sensitivity of the used detectors.

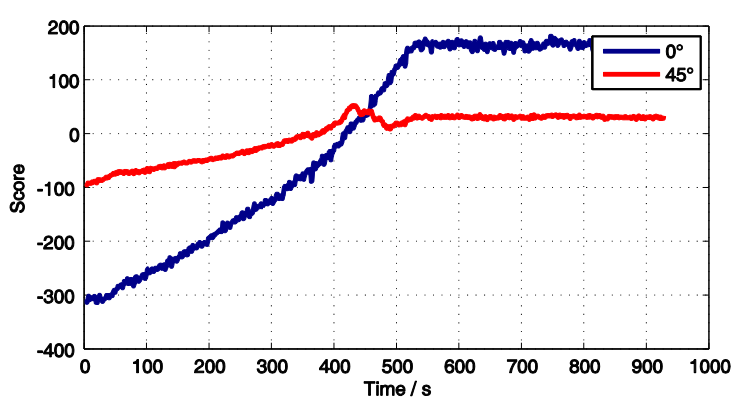

Figure 6: Transformed data (score, first principal component) of both data sets $\left(0^{\circ}, 45^{\circ}\right)$

The first principal components of each raw spectra contain the distinctive information of $\boldsymbol{X}$. In this case they contain the information about the moisture and the surface roughness. This is comparable to measurement results in figure 4 as well.
The first eigenvectors of the two data sets are almost identical. For larger thicknesses of the water layer there is no change of the upper boundary layer roughness. This leads to the assumption, that the influence of absorption effects in the layer must be equal for large layer thicknesses (at $0^{\circ}$ and $45^{\circ}$ ). In consequence the trend of the first principal components (Figure 6) must be equal. The factor between the curves is caused by the two not identical detectors: Gain and offset were adjusted to provide comparability. Figure 7 shows the matched curves referenced to their final value (dry surface).

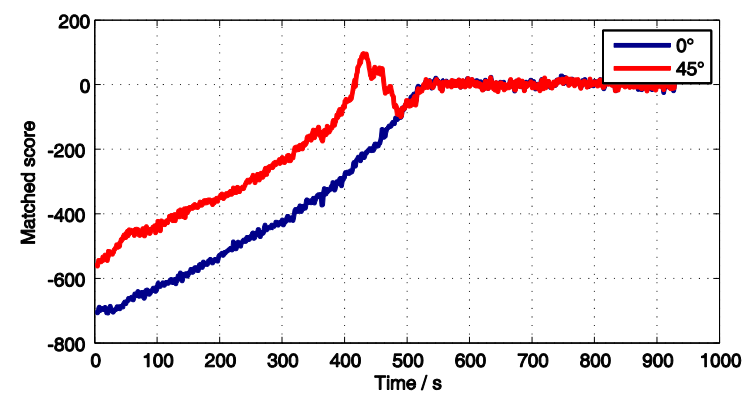

Figure 7: Matched principal components (scores)

Noticeable is the exact match of the two curves when the probe is dry (time $>500 \mathrm{~s}$ ). Resultant the difference between the two curves from figure 7 is caused by the specular reflected radiation shown in figure 8 .

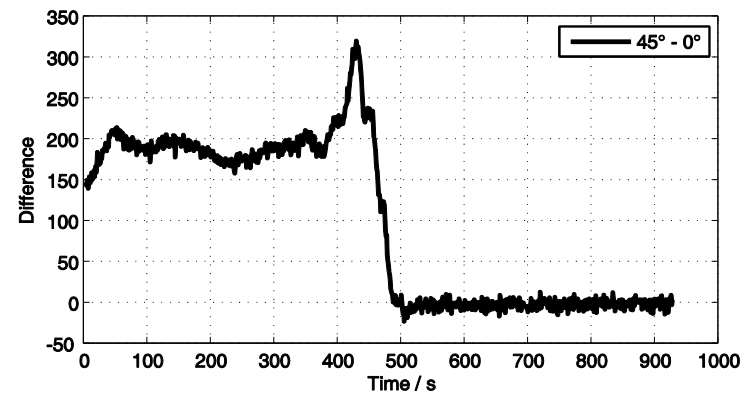

Figure 8: Difference between the matched principal components caused by specular reflected radiation

The detector signals are affected by specular and diffuse reflection. Here (Figure 8) the two influences are decoupled and only the specular reflected part is visualized. From the time at $500 \mathrm{~s}$ the specular reflected radiation disappears because the surface is dry. 


\section{Simulation}

A simulation is used to illustrate the occurring effects and should later (in further investigations) be used to identify the model parameters by an inverse approach. The simulation gives deeper insight into the complex drying process especially in the region of dull finish.

The irradiation at $45^{\circ}$ (according to DIN) causes multiple reflections in the film, which strongly depends on the roughness grade $\left(D_{01}, D_{12}\right.$, see Figure 9 ) and the reflectivity $\rho$ of boundary layers.

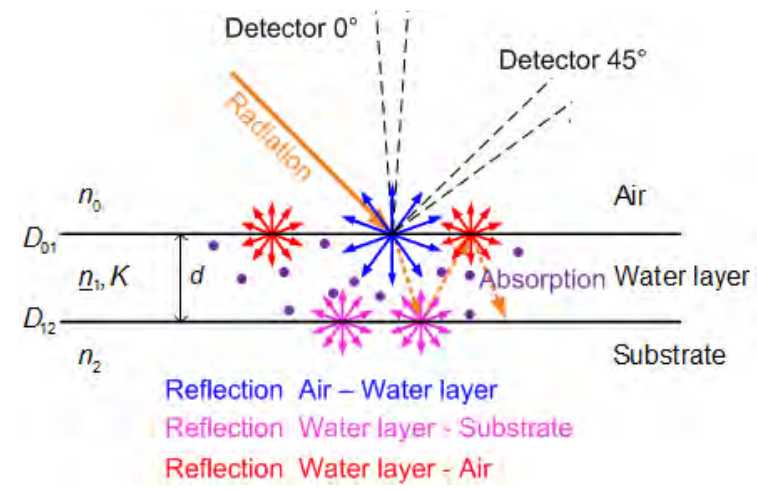

Figure 9: Parameter of simulation model

To describe the drying process by simulation, the following assumptions were made.

- No scattering in the water layer

- Ideal diffuse reflecting substrate (Lambertian reflector)

- No radiation absorption at the lower boundary layer (substrate)

- Water layer dries homogeneously

- Radiation is not polarized

For $D=1$ there is only diffuse reflection. If the roughness grade is $D=0$ only specular reflection occurs. In the following the diffuse radiation is always described as an ideal Lambertian reflector. Figure 10 shows the approach to describe a reflection on a rough surface $(0 \leq D \leq 1)$ with ideal specular and Lambertian reflectance.

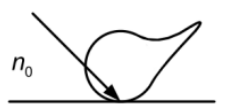

$n_{1}$

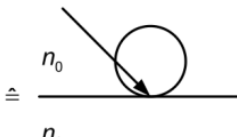

$n_{1}$

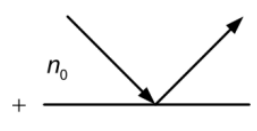

$n_{1}$
Figure 10: Combination of diffuse and specular radiation to describe the reflectance on a rough surface

The absorption in the layer is described by Lambert-Beer law. The reflectivity $\rho(\theta)$ at the boundary layer is calculated by Fresnel equations.

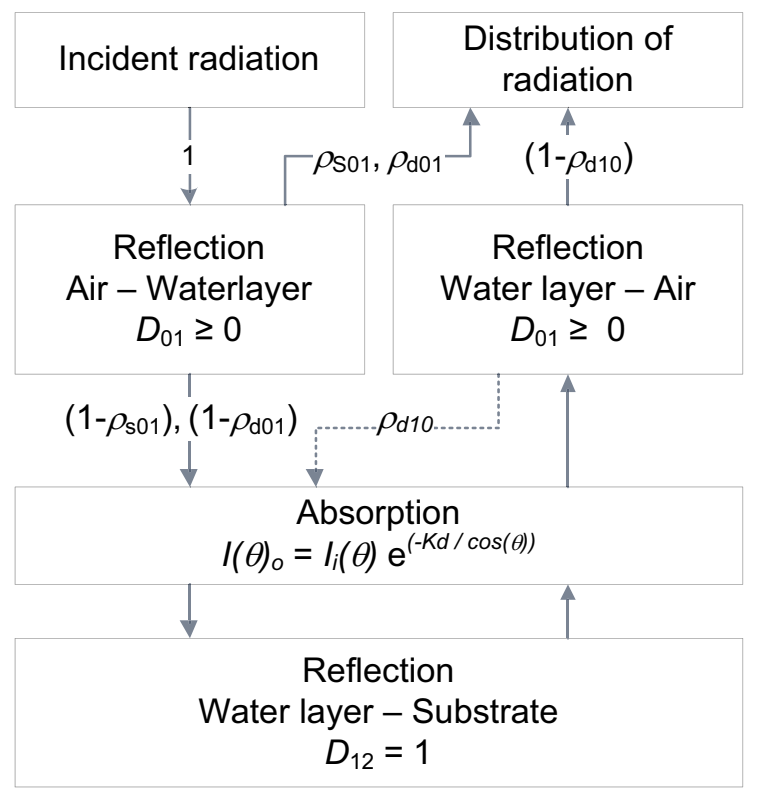

Figure 11: Program sequence of data processing (diffuse and specular reflectivity $\rho_{\mathrm{d}} \rho_{\mathrm{s}}$ )

Radiation components out of layer above $49^{\circ}$ cannot leave the layer because of total reflection. In the simulation the total reflected radiation is reflected back to substrate, where it is reflected with an ideal Lambertian distribution. This produces a kind of "lens effect", whereby the radiation parts at a detector angle of $0^{\circ}$ to $49^{\circ}$ increases.

This effect cannot be observed in the measurement result (Figure 3). Multiple reflections in the layer represent a lengthening of the optical path effectively. This produces a higher absorption in the layer and can be modeled by a larger layer thickness. For this reason the part of reflected radiation is not considered in the following simulation results. This missing diffuse reflected radiation is compensated by the roughness grade $D_{01}>0$ (even for plain surfaces).

The analysis with the PCA shows the qualitative influence of the specular reflected radiation at the boundary layers. The specular reflected radiation is influenced by the roughness grade $D_{01}$. Therefor the roughness grade $D_{01}$ results from the trend of Figure 8. The parameter for the film thickness $d$ follows from the tendency of Figure 9. 

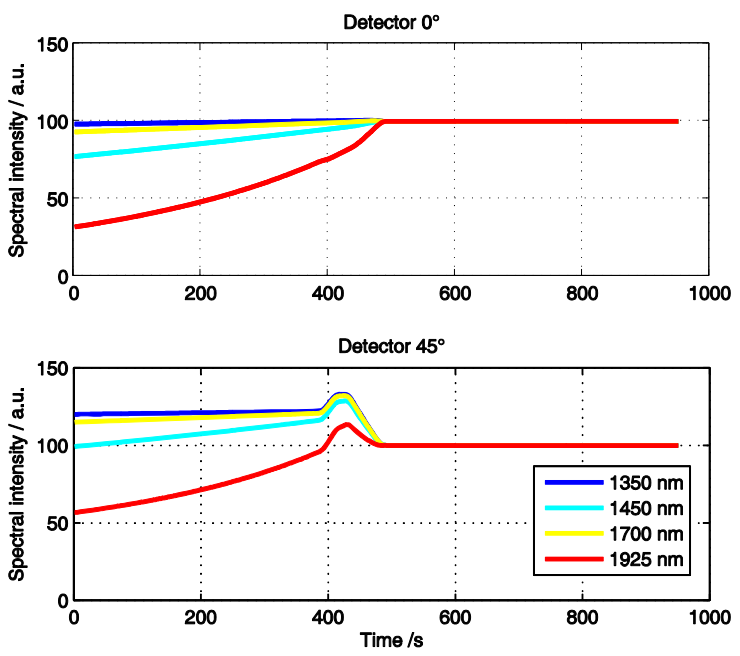

Figure 12: Simulation results at $0^{\circ}$ and $45^{\circ}$

The simulation results show good match to the measured data.

\section{Summary and outlook}

This investigation shows the significant influence of gloss on moisture measurement.

Using two detectors at $45^{\circ}$ and $0^{\circ}$ the detection of specular reflected radiation parts is possible. Both detected signals are influenced by diffuse and specular reflected radiation. The degree of coupling of both parts depends on the dullness (e. g. roughness) of substrate surface and the angle of the detector alignment.

The developed physical simulation model and the statistical analysis by PCA are suitable to separate both influences.

The future work aims at the development of algorithms to correct the influences on measurement values. This method offers a new and promising possibility to monitor drying processes particularly in the region of dull finish.

\section{References}

[1] Wetzlar, D.; Görlich V.: Messtechnische Bestimmung der Strahlungsabsorption in einer Schicht unter Berücksichtigung einer variablen Oberflächenstruktur, CMM Karlsruhe (2011)

[2] Kortüm, G.: Reflexionsspektroskopie: Grundlagen, Methodik, Anwendungen. Berlin: Springer (1969)

[3] Jolliffe, I.T.: Principal Component Analysis. New York: Springer (2002)

[4] Völz, H. G.: Industrielle Farbprüfung. Weinheim: VCH-Wiley (2001) 